PROCEEDINGS OF THE

AMERICAN MATHEMATICAL SOCIETY

Volume 126, Number 10, October 1998, Pages 3061-3064

S $0002-9939(98) 04614-0$

\title{
A NOTE ON FIXED POINT THEOREMS FOR SEMI-CONTINUOUS CORRESPONDENCES ON $[0,1]$
}

\author{
ZHOU WU
}

(Communicated by Palle E. T. Jorgensen)

\begin{abstract}
This paper presents a fixed point theorem for correspondences on $[0,1]$. Some examples comparing it to related work and also some simple applications to game theory are included.
\end{abstract}

\section{INTRODUCTION}

A function that is either continuous or nondecreasing from $[0,1]$ to itself has a fixed point. What are common points between these two results? This problem has been investigated independently by Milgrom and Roberts [4] and Guillerme [1]. Their findings can be summarized as:

Theorem 1.1. If a real function $f:[0,1] \rightarrow[0,1]$ is upper semicontinuous on the right and lower semicontinuous on the left, then it has a fixed point.

On the other hand, Strother [5] asserted that a continuous multivalued function on $[0,1]$ has a fixed point. This is a surprising result in the sense that a fixed point theorem for a multivalued function usually requires the function to have convex values - for example, Kakutani's theorem, or some theorems involving a continuous selection (for which Michael's [3] result is often needed). Do we have an analogy to Theorem 1.1 for multivalued functions (which are often called correspondences in economics)? The answer is positive. Strother's result was proved also by constructing a continuous selection. But our result is not. Moreover, we shall give an example to show that correspondences satisfying the hypothesis of our theorem may not allow a continuous selection. We also include some simple applications to game theory.

\section{Fixed POINT THEOREMS}

First we give some definitions. Let $T: \mathbb{R} \rightarrow 2^{Y}$ be a correspondence, where $\mathbb{R}$ is the real line, $Y$ is a metric space and $2^{Y}$ is the set of all nonempty subsets of $Y . T$ is said to be upper semicontinuous on the right (RUS) if for any $\bar{x} \in \mathbb{R}$ and any sequence $\left(x_{n}\right)$ such that $x_{n} \downarrow \bar{x}$, if a sequence $\left(y_{n}\right)$ converging to $\bar{y}$ is such that $y_{n} \in T\left(x_{n}\right) \forall n$, then $\bar{y} \in T(\bar{x}) ; T$ is said to be lower semicontinuous on the

Received by the editors September 3, 1996 and, in revised form, March 17, 1997.

1991 Mathematics Subject Classification. Primary 47H10, 54H25, 90D40; Secondary 26A15.

Key words and phrases. Fixed point, game theory.

The author would like to thank Professor S. Dasgupta for inspiring this problem, and Professor K. K. Tan for several discussions. 
left (LLS) if for any sequence $x_{n} \uparrow \bar{x}$ and every $\bar{y} \in T(\bar{x})$, there is a sequence $\left(y_{n}\right)$ converging to $\bar{y}$ such that for every $n$, one has $y_{n} \in T\left(x_{n}\right)$.

Now we are ready to prove an intermediate value theorem.

Proposition 2.1. Let $T:[0,1] \rightarrow 2^{\mathbb{R}}$ be an RUS and LLS correspondence. Suppose that $T([0,1])$ is contained in some bounded set of $\mathbb{R}$ and for any $y \in T(0)$ we have $y \geq 0$, while for any $y \in T(1)$ we have $y \leq 0$. Then there exists $x \in[0,1]$ such that $0 \in T(x)$.

Proof. Define $x_{L}=\inf \{x:$ there exists some $y \in T(x)$ such that $y \leq 0\}$. Obviously $x_{L}$ exists. If $x_{L}=1$, we have $0 \in T(1)$. Indeed, otherwise, take any $y_{L} \in T(1)$. Then $y_{L}<0$. Now take any sequence $\left(x_{n}\right)$ in $[0,1]$ such that $x_{n} \uparrow x_{L}$. Since $T$ is LLS, we can find $\left(y_{n}\right)$ such that $0 \leq y_{n} \in T\left(x_{n}\right)$ for each $n$ and $y_{n} \rightarrow y_{L}$. This is a contradiction. In fact, $T(1)=\{0\}$ in this case.

Now suppose that $0 \leq x_{L}<1$. Then there is a sequence $\left(x_{n}\right)$ in $[0,1]$ such that $x_{n} \downarrow x_{L}$ and there is a sequence $\left(y_{n}\right)$ such that, for each $n, y_{n} \in T\left(x_{n}\right)$ and $y_{n} \leq 0$. Since the set $T([0,1])$ is bounded, we can have a subsequence $\left(y_{n_{i}}\right)$ of $\left(y_{n}\right)$ converging to some $y_{L}=\lim y_{n_{i}} \leq 0$. Since $T$ is RUS, we have $y_{L} \in T\left(x_{L}\right)$. If $x_{L}=0$, since $y_{L} \in T\left(x_{L}\right)=T(0)$, we have $y_{L} \geq 0$. Thus $y_{L}=0$, which implies $0 \in T(0)$ in this case. If $x_{L}>0$, we can find a sequence $\left(x_{n}\right)$ in $[0,1]$ such that $x_{n} \uparrow x_{L}$. Since $T$ is LLS, there exists $\left(y_{n}\right)$ such that for each $n, y_{n} \in T\left(x_{n}\right)$ and $y_{n} \rightarrow y_{L}$. Since $y_{n}>0$ for each $n$, we have $y_{L} \geq 0$. So $y_{L}=0$, i.e. $0 \in T\left(x_{L}\right)$.

Theorem 2.2. Let $T:[0,1] \rightarrow 2^{[0,1]}$ be an RUS and LLS correspondence. Then $T$ has a fixed point.

Proof. Define $G:[0,1] \rightarrow 2^{\mathbb{R}}$ by $G(x)=\{y-x:$ for $y \in T(x)\}$; then $G$ satisfies all conditions of Proposition 2.1. So there is $\bar{x}$ such that $0 \in G(\bar{x})$, which means that $\bar{x} \in T(\bar{x})$.

Example 2.3. Let $F:[0,1] \rightarrow 2^{[0,1]}$ be defined by

$$
F(x)=\left\{\begin{array}{lc}
{[1 / 3-(x+1) / 8,2 / 3+(x+1) / 8],} & x<1 / 2 ; \\
{[1 / 3,2 / 3] \cup\{3 / 4\},} & x=1 / 2 ; \\
{[2 / 5,3 / 5] \cup\{3 / 4\},} & x>1 / 2 .
\end{array}\right.
$$

It is easy to check that $F$ is RUS and LLS, but it is neither upper semicontinuous nor lower semicontinuous. Moreover, it has non-convex values. Therefore the usual Kakutani fixed point theorem cannot be applied.

In [5], Strother proved the result we mentioned above by constructing a continuous selection of the continuous correspondence. We give an example to show that our conditions may not allow a continuous selection.

Example 2.4. Let $F:[0,1] \rightarrow 2^{[0,1]}$ be defined by

$$
F(x)= \begin{cases}{[0,1],} & x \leq 1 / 2 \\ \{|\sin (1 /(x-1 / 2))|,|\cos (1 /(x-1 / 2))|\}, & x>1 / 2 .\end{cases}
$$

Then $F$ is RUS and LLS, but it is not possible that $F$ has a continuous selection. For if it had, we denote it by $f$ and suppose $f(.5)=a \in[0,1]$. If $a=\sin (\pi / 4)=$ $\cos (\pi / 4)$, we take $x_{n}=.5+1 /(n \pi)$; then $F\left(x_{n}\right)=\{0,1\}$, and $d\left(a, F\left(x_{n}\right)\right)=$ $(1-\sin (\pi / 4))$. If $a \neq \sin (\pi / 4)$, take $x_{n}=1 / 2+1 /(n \pi+\pi / 4)$; then $d\left(a, F\left(x_{n}\right)\right)=$ $|a-\sin (\pi / 4)|$. So $F(x)$ has no continuous selection. This also demonstrates that $F$ 
is not continuous, for otherwise $F$ would have a continuous selection by Strother's Theorem.

Again, since $F$ is not convex-valued, the Kakutani fixed point theorem cannot be applied.

\section{Applications to game theory}

Consider a two-person game in which the two players are identified as Player 1 and Player 2. The strategy spaces for them are sets $X_{1}$ and $X_{2}$, respectively. The best responses of Player 1 and Player 2 are defined by correspondences $T_{1}: X_{2} \rightarrow$ $2^{X_{1}}$ and $T_{2}: X_{1} \rightarrow 2^{X_{2}}$, respectively. Let $T: X_{1} \times X_{2} \rightarrow 2^{X_{1}} \times 2^{X_{2}}$ be defined by $T(x)=T_{1}\left(x_{2}\right) \times T_{2}\left(x_{1}\right)$ for $x=\left(x_{1}, x_{2}\right) \in X_{1} \times X_{2}$. The set of Nash equilibrium points of this game is defined as the set of fixed points of $T$. Note that such a game has a non-empty set of equilibria if and only if the composite correspondence $T_{1} T_{2}: X_{1} \rightarrow 2^{X_{1}}$ or $T_{2} T_{1}: X_{2} \rightarrow 2^{X_{2}}$ has a fixed point.

Theorem 3.1. For a two-person game on $[0,1] \times Y$, where $Y$ is a compact metric space, let $T_{1}(y)$ be the best response of Player 1 and $T_{2}(x)$ be the best response of Player 2. We suppose that $T_{1}$ is continuous with closed values and $T_{2}$ is RUS and LLS with closed values. Then there is a Nash equilibrium for this game.

Proof. We define $T:[0,1] \rightarrow 2^{[0,1]}$ by $T=T_{1} T_{2}$. If $T$ has a fixed point $\hat{x}$, then there is $\hat{y} \in T_{2}(\hat{x})$ such that $\hat{x} \in T_{1}(\hat{x})$. So $(\hat{x}, \hat{y})$ will be a Nash equilibrium of the game.

Now we prove that $T$ is RUS. Suppose that $x_{n} \downarrow x$ and $z_{n} \in T\left(x_{n}\right) \rightarrow z$. Then there is $y_{n} \in T_{2}\left(x_{n}\right)$ such that $z_{n} \in T_{1}\left(y_{n}\right)$. Since $Y$ is compact, without loss of generality we suppose that $\left(y_{n}\right)$ converges to $y$. Then $z \in T_{1}(y)$, since $T_{1}$ is continuous with closed values (hence it is closed). But $y \in T_{2}(x)$, since $T_{2}$ is RUS. Therefore $z \in T_{1} T_{2}(x)=T(x)$. That is, $T$ is RUS.

Next we shall prove that $T$ is LLS. Suppose that $x_{n} \uparrow x$ and $z \in T(x)$. First there is $y \in T_{2}(x)$ such that $z \in T_{1}(y)$. Since $T_{2}$ is LLS, there is $y_{n} \in T_{2}\left(x_{n}\right)$ such that $y_{n} \rightarrow y$. Since $y_{n} \rightarrow y$ and $z \in T_{1}(y)$, there is $z_{n} \in T_{1}\left(y_{n}\right)$ such that $z_{n} \rightarrow z$, since $T_{1}$ is lower semicontinuous. Now we have $z_{n} \in T_{1} T_{2}\left(x_{n}\right)$ such that $z_{n} \rightarrow z \in T(x)$. Thus $T$ is LLS.

So by Theorem 2 , there is a fixed point $\hat{x} \in T(\hat{x})$, i.e. there is a Nash equilibrium for the two person game.

Corollary 3.2. For a two-person game on $[0,1] \times Y$, where $Y$ is a Banach space, let $T_{1}(y)$ be the best response of Player 1 and $T_{2}(x)$ be the best response of Player 2. We suppose that $T_{1}$ is lower semicontinuous with closed convex values and $T_{2}$ is RUS and LLS with closed values. Then there is a Nash equilibrium for this game.

Proof. In fact, by Theorem 3.2" in Michael [3] there is a continuous selection of $T_{1}$. So the result follows.

\section{REFERENCES}

1. J. Guillerme, Intermediate value theorems and fixed point theorems for semi-continuous functions in product spaces, Proc. Amer. Math. Soc. 123, 2119-2122(1995). MR 95i:54053

2. E. Klein and A. C. Thompson, Theory of Correspondence: including applications to mathematical economics, John Wiley \& Sons, 1984. MR 86a:90012

3. E. Michael, Continuous selections. I. Ann. of Math. 63 361-382(1956). MR 17:990e 
4. P. Milgrom and J. Roberts, Comparing equilibria, Amer. Econ. Rev. 84, 441-459(1994).

5. W. L. Strother, On an open question concerning fixed points, Proc. Amer. Math. Soc.4, 988993 (1953). MR 15:642c

Department of Mathematics, Statistics \& Computing Science, Dalhousie University, Halifax, Nova Scotia, Canada B3H 3J5

Current address: Faculty of Computer Science, Daltech, Dalhousie University, P.O. Box 1000, Halifax, Nova Scotia, Canada B3J 2X4

E-mail address: zwu@cs.dal.ca 